\title{
Editorial: \\ Didaktik(en) zwischen \\ Kontinuität und \\ Neuorientierung
}

\section{Peter Sieber und Thérèse Thévenaz}

Aus verschiedenen Gründen stehen heute Konstituierung und Positionierung der Didaktik/en im Zentrum des Interesses. Diskussionen und Anstrengungen für eine Neustrukturierung der Lehrer/innenausbildung bedingen ein Überdenken der Funktionen und Aufgaben der Didaktik. Neue Konzepte und Theorien, die während den vergangenen Jahrzehnten in den Fachdidaktiken entwickelt wurden, stehen dafür zur Verfügung. Wichtige Entwicklungen und Vorgaben entstehen auch auf bildungspolitischer Ebene, zum Beispiel mit dem Projekt Fachdidaktiken ${ }^{1}$ in der Schweiz-Gezielter Kompetenzaufbau: Qualifizierung von Lehrerinnen und Lehrern und Etablierung der Forschung (Projekt Fachdidaktik, 2005), dessen Bericht momentan in der Vernehmlassung ist. Vor diesem Hintergrund befassen sich der thematische Teil sowie die Rubrik Diskussion dieser Ausgabe intensiv mit Fragen des Aufbaus und der Positionierung dieses Fachgebietes.

Im thematischen Teil der vorliegenden Nummer der Schweizerischen Zeitschrift für Bildungswissenschaft legen wir den Schwerpunkt auf die Entwicklung der Didaktiken in den letzten zwanzig Jahren; gleichzeitig knüpfen wir an die zwei Nummern von Bildungsforschung und Bildungspraxis ${ }^{2}$ aus den Jahren 1990/91 an, in denen dieses Thema bereits behandelt wurde. Wir zeigen sowohl die einheitlichen als auch die divergierenden Hauptlinien der Fachdidaktiken in deutsch- und französischsprachigen Gebieten auf, aus deren je spezifischer wissenschaftlicher Kultur sie hervorgegangen sind.

Mit dieser Perspektive beabsichtigten wir als Erstes, den Dialog über den wissenschaftlichen Umgang mit zwei zentralen Fachdidaktiken in deutsch- und französischsprachigen Gebieten in Gang zu bringen und herauszuarbeiten, welche Entwicklungslinien sich historisch betrachtet ergeben: Bildeten die Mathematikdidaktik und die Erstsprachdidaktik (deutsch oder französisch, je nach Sprachgebiet) das Fundament des obligatorischen Volksschulunterrichts? Um einen konstruktiven Dialog in Bewegung zu setzen, haben wir Autoren gebeten, die Entwicklungsschritte der beiden Didaktiken innerhalb ihrer eigenen Sprachkultur zu skizzieren. 
Mit der Präsentation wichtiger Entwicklungslinien der Didaktikforschung richten wir einen zweiten Fokus auf Didaktiken, die erst in jüngerer Zeit thematisiert worden sind. Es handelt sich einerseits um die Didaktiken der Kunst und Musik, andererseits um die Vergleichende Didaktik, die - wie die Leserin und der Leser in der vorliegenden Zeitschriftennummer feststellen können - nicht auf einer allgemeinen Didaktik basiert.

Von den neueren Fachdidaktiken hätte man an Stelle der Kunstdidaktik genauso gut die Turn- und Sportdidaktik wählen können. Wir haben uns für einen anderen Fachbereich entschieden, weil im Teil Varia der Nummer 3/2003 bereits ein Beitrag über die Turn- und Sportdidaktik erschienen ist.

Als Kontrastpunkt zum neuen Arbeitsfeld der Vergleichenden Didaktik hätten wir gerne einen Beitrag aus der Sicht der Allgemeinen Didaktik präsentiert, der die starke Verankerung der Didaktik in der Lehrer/innenausbildung und der Erziehungswissenschaft im deutschsprachigen Raum gezeigt hätte. In ihrer Eigenschaft als differenzierte, kritische Theorie der Ausbildung bildet die Allgemeine Didaktik Kategorien, um die Lehrinhalte, die Methoden und den Lernprozess von ihrer ideologischen und gesellschaftlichen Wirkung her betrachten zu können. Ein Beitrag der Allgemeinen Didaktik hätte in der Zusammenführung der wichtigsten Entwicklungslinien die gegenwärtige bildungspolitische Situation kritisch beleuchten können.

Ausgehend von dieser Problemstellung haben wir uns an B. Schneuwly mit der Bitte um einen Diskussionsbeitrag gewandt. Drei Gründe haben uns zu dieser Wahl bewogen: Erstens ist Schneuwly seit 1997 Inhaber einer der - in der Schweiz sehr seltenen - Lehrstühle für Fachdidaktik, genauer gesagt für Französischdidaktik. In dieser Eigenschaft und durch seine institutionelle Einbindung vertritt er den Standpunkt des Didaktikers. Ausserdem war er massgeblich an der Herausgabe der beiden Nummern der Zeitschrift Bildungsforschung und Bildungspraxis (3/1990; 1/1991) zu den Themen Didaktik und Fachdidaktik beteiligt und deshalb eine geeignete Person, um über Entwicklungsverläufe in diesen Gebieten zu schreiben. Schliesslich wirkt B. Schneuwly als Experte der Romandie im Rahmen des Projekts „Fachdidaktiken in der Schweiz» (Projekt Fachdidaktik, 2005) in der Arbeitsgruppe für eine schweizerische „Graduiertenschule für Fachdidaktik» an bildungspolitischen Diskussionen mit. Eine solche Graduiertenschule könnte Doktorandinnen und Doktoranden mit Interesse an einer Dissertation in Didaktik betreuen und sowohl Forschung wie Nachwuchsförderung an Universitäten und Pädagogischen Hochschulen sichern helfen.

Die Rubrik Diskussion hat hier zum Zweck, die Beiträge des thematischen Teils im Licht des heutigen Standes der wissenschaftlichen Forschung und deren institutioneller Verankerung zu sehen sowie auf die offenen Problemstellungen in Forschung und Lehrer/innenausbildung im Rahmen der Tertiarisierung hinzuweisen. 
Wir wollen es vorweg nehmen: Unser Vorhaben ist nur teilweise gelungen, aber wir hoffen, dass die vorliegende Nummer Anregung für weitere Beiträge sein wird. Wir haben bis zum Zeitpunkt des In-Druck-Gehens dieses Heftes leider keinen Beitrag aus der Perspektive der Allgemeinen Didaktik erhalten. Die Restrukturierung der Forschung und der Ausbildung in der (Deutsch-)Schweiz scheint die wenigen Ressourcen im Bereich Didaktik völlig zu absorbieren.

Die beiden Beiträge betreffend Erstsprachdidaktik, einerseits zur Didaktik des schriftlichen Ausdrucks (Bronckart, in dieser Nummer), andererseits zur Didaktik des Schreibens ( Sieber, in dieser Nummer) stellen den Anfang eines Dialogs über die Entwicklung der Forschung und der Problemstellungen in einem vergleichbaren Gebiet während einer vergleichbaren Zeitspanne dar. Mit der gleichen Absicht sind wir mit unseren Vorstössen bei der Mathematikdidaktik in der Deutschschweiz und in Deutschland auf taube Ohren gestossen. Die fehlenden Reaktionen im Bereich der Mathematikdidaktik erklären wir uns sowohl durch die stattfindende Reorganisation der Lehrer/innenausbildung in den Pädagogischen Hochschulen als auch durch die fachliche Fokussierung unserer Zeitschrift auf Erziehungswissenschaften.

Diese Situation zeigt eines deutlich: Durch die Tertiarisierung der Lehrer/innenbildung wird eine Neudefinition der Forschung in den pädagogischen Hochschulen und den Universitäten unumgänglich. Die Umstrukturierung erfolgte erst kürzlich und so blieb für interessierte Wissenschafter wenig Kapazität, sich mit den Entwicklungen ihres Gebietes auseinander zu setzen und dies zu publizieren. Zudem verfügen jene Didaktiker, die institutionell in den Universitäten eingebunden sind, über eigene Publikationsorgane und haben daher eher wenig Interesse, in einer Zeitschrift der Erziehungswissenschaft zu publizieren. Für die Entwicklung der Didaktiken - nicht nur der Mathematikdidaktik - bleibt aber die Frage der institutionellen Einbindung wichtig. In dieser Hinsicht ist es beachtenswert, dass die Autoren der Beiträge dieser Nummer sowohl in der Lehrer/innenausbildung tätig sind - was in der Fachdidaktik und in der Allgemeinen Didaktik nicht neu ist - als auch in den Human- und Sozialwissenschaften, d.h. in den fachspezifischen Bereichen und/oder in den Erziehungswissenschaften.

In dieser Situation haben wir uns aus der Fülle von Aspekten der Entwicklung des Feldes der Didaktiken auf drei beschränkt. Ein weiterer Gesichtspunkt soll Stoff für künftige Diskussionen liefern: die Positionierung der Fachdidaktiken - auch innerhalb der Institutionen - im Spannungsfeld der Erziehungs- und Sozialwissenschaften, der Fachwissenschaften und der Schule (Fachkommission Fachdidaktik, 1998; Projekt Fachdidaktik, 2005). Hier stehen drei Aspekte im Vordergrund:

1. Bildungsstandards und geforderte Kompetenzen der Schülerinnen und Schüler

2. Schulisches Curriculum und die Progression der Lehrinhalte

3. Die aktuelle Einschätzung des didaktischen Dreiecks von Schüler/in, Unterrichtsgegenstand und Lehrperson 
1. Die Debatte über den Einfluss des Lehr/Lernprozesses in der Schule und über die Fähigkeiten der Schüler/innen am Ende der obligatorischen Schulzeit ist von grosser Aktualität. Sie thematisiert die Interventionsmöglichkeiten der Didaktiken. Sind diese in der Lage, die nötigen Leitlinien und Lehr/Lerninhalte zu bieten, um den politischen und gesellschaftlichen Forderungen gerecht zu werden? Die aktuellen Diskussionen gehen von einer Entwicklung von Bildungsstandards aus, die eine Evaluation der Schüler/innenleistungen überhaupt erst ermöglichen und die Kompetenzniveaus durch eine genaue Definition testbar machen. Wie gängig sind die Begriffe „Standards» und „Kompetenzen» im Bereich der Didaktiken und wie werden sie eingesetzt? Wie gestaltet sich der Aufbau von Kompetenzen, die grösstenteils über das Resultat und die Fähigkeit, situationsgerecht zu handeln, definiert werden? Wie geht die Progression der Lehr/Lerninhalte dabei vor sich? Wenn wir davon ausgehen, dass alles, was getestet werden kann, irgendwann gelehrt worden ist - welches Curriculum bestimmt dann die Wahl der Testaufgaben und des Settings? Die beiden letzten Fragen betreffen den zweiten der oben erwähnten Aspekte.

2. Die polit-ökonomischen und gesellschaftlichen Erwartungen im Bildungsbereich zeigen sich nicht allein in Bezug auf Fragen nach Auswirkung und Ergebnis, sondern auch durch die Forderung nach Vereinheitlichung und Systematik der Bildungsinhalte, die künftigen Generationen vermittelt werden sollen. Diesen Erwartungen wird man nur gerecht mit einem sorgfältig gewählten Lehrplan, einer systematischen Gestaltung der Inhalte, die zu einer guten praktischen Umsetzung führen (Westbury, 1998). Dazu bestimmt, Orientierung für die Praxis der Lehrpersonen zu gewährleisten, muss ein Curriculum auch die Konzepte liefern, die sich in die Praxis umsetzen lassen: in Aufgabenstellungen, in Situationen und auch in gut organisierten, kohärenten Unterrichtssequenzen, damit die Schülerinnen und Schülern jene Fähigkeiten erwerben können, die während deren ganzer Schulzeit von ihnen erwartet werden. Dazu muss das Curriculum konsequenterweise die Inhalte nach einem Zeitplan in progressive Stufen einteilen und die wichtigsten Lernschritte bereits einplanen und optimal staffeln. Die didaktische Forschung kann sich einer Reflexion über den Aufbau des Lehrplans über die gesamte Schulzeit nicht entziehen. Gehen die Beiträge in dieser Nummer auf irgendeine Art und Weise auf diese Überlegungen ein?

3. Seit ihrer Entstehung in den 1970/80er Jahren lassen sich die Fachdidaktiken im System des Didaktischen Dreiecks von Schüler/in, Lehrperson und Gegenstand verorten. $\mathrm{Zu}$ verschiedenen Zeiten und in unterschiedlichen Konzepten erhielt jeweils eine der Achsen mehr Gewicht, sei es die Achse Inhalt-Schüler/in oder die Achse Inhalt-Lehrperson oder auch die von Lehrperson und Schüler/in. Schaut man, welche Achsen bevorzugt werden, wird das didaktische Dreieck zu einer weiteren, einer dritten Beobachtungsmöglichkeit der Weiterentwicklung der Didaktik. Sind wir Zeugen einer Verschiebung der Achsen? Wenn ja, welcher? 
Die Weiterentwicklung, die wir umreissen können, verweist auf eine Verschiebung von den Progammreformen, von einer innovativen Inhaltsentwicklung hin zu Konzeptualisierungen, die einen Bruch mit der innovationsorientierten Bildungsreform darstellen. Manche Didaktiken interessieren sich für den alltäglichen Ablauf des Lehr/Lernprozesses eines gegebenen Lehrinhalts und die Analyse der allgemeinen und spezifischen Regeln ( Margolinas; Schubauer-Leoni \& Leutenegger, in dieser Nummer). Über die Bindung der französischsprachigen und der deutschsprachigen Didaktiken an ihre jeweilige Kultur hinaus interessieren sich diese Konzeptualisierungen der Didaktik für Adaptationen aus verschiedenen akademischen Fächern für die Erfordernisse und Regelungen des Lehr/Lernprozesses eines gegebenen Stoffes. Diese neue Bewegung der Anleihen und Überarbeitungen von Inhalten aus den verschiedenen Fachbereichen wird durch die Perspektive auf die Lehr/Lern-Prozesse kanalisiert; dies klingt in allen Beiträgen dieser Nummer an. Die Theorie zu diesem Phänomen der Didaktik als Ganzem findet sich im Beitrag Schubauer-Leoni und Leutenegger mit dem Titel „Une relecture des phénomènes transpositifs à la lumière de la didactique comparée». Nach ihrer Meinung ist der strikte Applikationismus - die direkte Umsetzung von Wissensbeständen der Fachwissenschaften in Gegenstände des Unterrichts - am Verschwinden, weil die Übernahmen aus den Fachwissenschaften unter didaktischen Perspektiven erfolgen und damit einer Transformation unterliegen.

Der Bezug auf Kompetenzen veranschaulicht diese didaktikspezifische Perspektivierung. Der Begriff Kompetenz wird in den Beiträgen zur Sprachdidaktik entweder beansprucht oder abgelehnt - die Debatte jedoch erst am Rande geführt. Fachkompetenz, ein Begriff aus der Arbeitswelt, mit dem Situationsbezogenheit und Effizienz angesteuert wird (Bronckart \& Dolz, 2000; Bulea \& Bronckart, 2005), kann ganz unterschiedlich verstanden werden. Er kann transversal oder disziplinär gefasst werden, eine bereits vorhandene Ressource des Akteurs bezeichnen oder eine erst durch eigenes Tun in einem dynamischen Prozess aktivierte Ressource meinen. Die Definitionen der Kompetenz ${ }^{3}$, von denen das Projekt Harmonisierung der obligatorischen Schule (HARMOS) (EDK, 2004) gemäss dem Prinzip der Bildungsfähigkeit ausgeht, beschreibt die Ressourcen, die eine Person entweder schon hat oder die sie erwerben kann. Kompetenz wird in voneinander abgrenzbaren Bereichen auf verschiedenen Niveaus beschrieben. HARMOS definiert Kompetenzen von den Grundlagen der Schulfächer aus und mit Bezug zu diesen. Um sie messbar zu machen, werden Kompetenzen in Aufgabenstellungen und Problemsituationen umgesetzt. Dieser Gesichtspunkt betrifft ohne Zweifel die Didaktiken, da diese sich der Aufgabe widmen, Aufgabenstellungen, Situationen, das Umfeld und darüber hinaus die Lehr/Lernsequenzen zu untersuchen.

Rückt dabei die Curriculumdebatte in den Hintergrund? Der Beitrag von Sieber in dieser Nummer evoziert dies, da er die Erforschung und Revision der Lehrpläne einer vergangenen Periode zuordnet. Von einer Orientierung am In- 
put sei man zu einer Orientierung am Output übergegangen oder eher zum Outcome, d.h., nicht allein zu den Ergebnissen, sondern ebenso zu den gesamten Auswirkungen des Lehrens und Lernens. Weitere Beiträge setzen einen anderen Schwerpunkt mit einer Perspektive, die Aufgaben und Situationen und die Untersuchung der Lernbedingungen der Schüler fokussiert. Damit wird eine Vertiefung der Kenntnisse über das Zusammenspiel von curricularen Anforderungen und dem unterrichtlichen Setting angestrebt. Darf die Didaktik sich der wichtigen Aufgabe entziehen, Vorstellungen über die Progression des Lernens zu entwickeln, und es verpassen, die Auswirkungen dieses Zusammenspiels im Schreiben, in der Mathematik oder im Musikhören zu untersuchen? Die Lerninhalte der Volksschule im Zusammenhang zu sehen ist eine wichtige Perspektive, die aber aktuell noch sehr programmatisch bleibt (siehe Dolz \& Schneuwly, 1996 oder für die 4. bis 8. Klasse: Saada-Robert, Claret Girard, Veuthey \& Rieben, 1997). Auch wenn Aufgaben, Situationen oder Sequenzen so ausgearbeitet sind, dass sie die Prozesse der Lehr/Lernaktivitäten in ihrer ganzen Komplexität zu integrieren vermögen, so stellt das Zeitmanagement der durch die Aufgaben, Situationen oder Sequenzen operationalisierten Inhalte doch eine Herausforderung für die Didaktiken dar, der sie sich bisher noch nicht wirklich gestellt haben. Die Mathematikdidaktik versteht das Zeitmanagement als Teil der Bewältigung einer Situation (Margolinas, in dieser Nummer). Daraus ergibt sich die Frage, auf welchen Konzepten die Einteilung des Zeitablaufs beruht, die sowohl die Aufteilung der allgemeinen Lehr/Lernziele auf die verschiedenen Schulstufen der obligatorischen Schulzeit (stufenübergreifende Progression), als auch das Zeitmanagement in Bezug auf Ziele und fachliche Inhalte innerhalb einer Stufe (Progression innerhalb einer Stufe) regelt. Wie weit berücksichtigt die von HARMOS angestrebte Definition von Kompetenzen solche Überlegungen? - Diese Frage betrifft alle Fachdidaktiken.

Was das didaktische Dreieck betrifft, beziehen sich die Beiträge darauf - meist mit der Favorisierung einer seiner Achsen. Den Einstieg im redaktionellen Teil macht Sieber mit seinem Beitrag, der sich auf die Achse Schüler/in - Schreiben von Texten mit all seinen komplexen Komponenten konzentriert. Er ist der einzige Autor in dieser Nummer, der explizit vom Lernenden und vom Lehr/Lerninhalt ausgeht und Textkompetenz fordert, die zu ermöglichen Pflicht der Schule sei. Der Beitrag von Margolinas zeigt, dass diese Achse seit den 1980er Jahren favorisiert wurde. Doch zur Erforschung des didaktischen Settings hat der Mathematikdidaktiker die Lehrerrolle übernommen, damit er sich so auf die Achse Schüler/Gegenstand konzentrieren kann. Diese Übernahme der Verantwortung hat zu einer Verschiebung des Gleichgewichts in Richtung Lehrer/Gegenstand geführt. Aus ähnlichen Gründen folgt die Französischdidaktik derselben Linie. Es geht darum, das Lehrerhandeln zu erforschen, d.h. zu beobachten, welche Bedingungen durch die Lehrperson bestimmt sind, wo Sachzwänge im Unterricht selber liegen, und welche Inhalte während der Schulstunde vermittelt 
werden. Dafür greift die didaktische Forschung auf empirische Beobachtungsmethoden zurück: einerseits aus der beschriebenen Sicht der didaktischen Intervention (Mili \& Rickenmann; Bronckart), andererseits, indem sie die Ausarbeitung des Lehrstoffs der Lehrperson überlässt, wie z.B. in der Untersuchung der alltäglichen Unterrichtspraxis ( Margolinas; Schubauer-Leoni \& Leutenegger ). Hier ist ein neuer Forschungsansatz entstanden, der mit der starr logisch aufgebauten Intervention bricht und sich seitdem öffnet und kontrovers diskutiert wird.

Die verschiedenen thematischen Beiträge skizzieren - unter den drei ausgewählten Aspekten - ein sehr breites Feld. Dieses Feld zu charakterisieren, Widersprüche und Gemeinsamkeiten zu kennzeichnen und daraus Perspektiven für die Weiterentwicklung herauszuarbeiten: Dieser Aufgabe widmet sich der Diskussionsbeitrag von B. Schneuwly. Es ist zu hoffen, dass damit die notwendige Diskussion eröffnet - und nicht abgeschlossen ist.

\section{Anmerkungen}

1 Es ist zu beachten, dass der Ausdruck Fachdidaktiken in der Übersetzung didactiques spécialisées von Didaktikern der französischsprachigen Gebiete als nicht adäquat angesehen wird und eine Aufgliederung in Allgemeine Didaktik und Fachdidaktik vorgibt.

2 Siehe die Nummern 3/1990 und 1/1991 von Bildungsforschung und Bildungspraxis .

3 „Sie (die Bildungsstandards, PS/TT) benennen erstens die Kompetenzen, welche die Schule ihren Schülerinnen und Schülern vermitteln muss, damit bestimmte zentrale Bildungsinhalte erreicht werden, und legen zweitens fest, welche Kompetenzen Kinder und Jugendliche bis zu einer bestimmten Jahrgangsstufe erworben haben sollen. Drittens sind die zu erreichenden Kompetenzen so konkret beschrieben, dass sie in Aufgabenstellungen umgesetzt und im Prinzip mit Hilfe von Testverfahren erfasst werden können» (EDK, 2004, S. 7).

\section{Literaturverzeichnis}

Bronckart, J.-P. \& Dolz, J. (2000). La notion de compétence: quelle pertinence pour l'étude de l'apprentissage des actions langagières. In J. Dolz \& E. Ollagnier (Hrsg.), L'énigme de la compétence en éducation (Raisons Éducatives n 2, S. 27-44). Bruxelles: De Beock.

Bulea, E. \& Bronckart, J.-P. (2005). Coda: pour une approche dynamique des compétences (langagières). In J.-P. Bronckart, E. Bulea \& M. Pouliot (Hrsg.), Repenser l'enseignement des langues: comment identifier et exploiter les compétences (S. 193-227). Villeneuve d'Ascq: Presses universitaires du Septentrion.

Dolz, J. \& Schneuwly, B. (1996). Genres et progression en expression orale et écrite. Eléments de réflexions à propos d'une expérience romande. Enjeux, 37/38, 49-75.

Eidgenössische Erziehungsdirektorenkonferenz (EDK) (2004). HarmoS - Zielsetzungen und Konzeption des Projekts, Juni 2004. Bern: EDK.

Fachkommission Fachdidaktik (1998). Impulse für die Fachdidaktik in der Schweiz. Beiträge zur Lehrerbildung (BzL), 16/2, 222-230.

Projekt Fachdidaktik (2005). Fachdidaktik in der Schweiz-gezielter Kompetenzaufbau: Qualifizierung von Dozierenden und Etablierung der Forschung. Schlussbericht. September 2005. Bern: EDK/CRUS/SKPH. 
Saada-Robert, M., Claret Girard, V., Veuthey, C. \& Rieben, L. (1997). Situations didactiques complexes et spécifiques pour l'entrée dans l'écrit en 1E-2E-1P-2P. Genève: FPSE et Maison des Petits.

Westbury, I. (1998). Didaktik and Curriculum Studies. In B. Gundem \& S. Hopmann, Didaktik and/or Curriculum: an International Dialogue (pp. 47-78). New York: Peter Lang. 


\section{Editorial: \\ Didactique(s) - entre continuité et réorientation}

\section{Peter Sieber et Thérèse Thévenaz}

Pour différentes raisons, les questions de constitution et de positionnement de la ou des didactique(s) sont aujourd'hui brûlantes: la discussion sur et les efforts pour une nouvelle structuration de la formation des maitresSES exigent une réflexion des fonctions et des tâches des didactiques. Ces réflexions sont soutenues par des développements conceptuels et théoriques - produits au cours des dernières décennies - dans les didactiques disciplinaires. Des orientations d'importance se profilent également au niveau de la politique de la formation à partir du projet - le rapport est actuellement en consultation - Didactiques spécialisées ${ }^{1}$ en Suisse - Constructions de compétences ciblées: qualification des professeurEs et implantation de la recherche (Projekt Fachdidaktik, 2005). De multiples raisons donc pour s'interroger sur les questions de constitution et de positionnement du champ dans la partie thématique et dans la rubrique discussion du présent numéro de la revue.

Dans les limites de la partie thématique de la Revue Suisse des Sciences de l'Éducation, nous avons souhaité mettre en perspective le développement des didactiques sur les vingt dernières années et faire ainsi écho aux deux numéros d'Education et Recherche ${ }^{2}$ parus sur la question dans les années 1990 . Nous avons projeté de montrer les principales directions, convergentes et divergentes, adoptées par les didactiques des disciplines émanant de la culture scientifique germanophone et francophone.

Dans cette perspective, nous aurions, premièrement, voulu mettre en dialogue la culture germanophone et francophone de deux didactiques disciplinaires phares, à savoir celle qui a été la première des didactiques à se revendiquer d'un nouveau champ de recherche, la didactique des mathématiques, et la didactique de la langue première d'enseignement, le socle de l'enseignement public obligatoire, le français ou l'allemand, selon les régions linguistiques. En demandant aux auteurs de tracer l'évolution de ces deux didactiques dans chacune des cultures, une mise en dialogue était possible.

Secondement, pour tracer quelques grandes lignes du développement de la recherche didactique, nous nous sommes tournés vers des didactiques émergentes, 
d'un côté, celles des arts plastiques et de la musique et, d'un autre côté, la didactique comparée qui, comme le lecteur ou la lectrice le découvrira dans le présent numéro, ne se revendique pas d'une didactique générale.

Concernant les didactiques disciplinaires émergentes, la didactique de l'éducation physique aurait pu être candidate au même titre que la didactique des arts. Comme une contribution est déjà parue dans la partie Varia du numéro 3/2003, nous avons décidé de privilégier un autre domaine disciplinaire émergent.

En contraste avec le développement du nouveau chantier que représente la didactique comparée, nous espérions publier une contribution du point de vue de la didactique générale, bien ancrée dans la culture germanophone de la formation des enseignants et des sciences de l'éducation. En tant que théorie critique plurielle sur la formation comme facteur d'émancipation, la didactique générale propose des catégories pour définir les contenus, les méthodes et l'apprentissage du point de vue de leurs effets idéologiques et sociaux. Ce discours didactique général portant sur la formation de la génération future aurait donné, en même temps que les lignes de développement du champ, un éclairage sur l'actualité de la politique éducative.

Dans une même perspective, nous nous sommes adressés à B. Schneuwly pour une contribution sous forme de discussion. Ce choix s'est imposé pour trois raisons: tout d'abord, alors que de telles chaires sont extrêmement rares en Suisse, il occupe depuis 1997 une chaire de didactique disciplinaire, plus précisément de didactique du français. A ce titre, par son ancrage institutionnel, il représente un point de vue de didacticien. Ensuite, jadis directement impliqué dans l'édition des deux numéros d'Education et Recherche sur les didactiques disciplinaires et la didactique, il se trouve bien placé pour traiter de leur évolution. Pour finir, dans le cadre du "Projet didactiques spécialisées en Suisse», B. Schneuwly est directement impliqué dans les discussions de politique de la formation en tant qu'expert romand du groupe de travail pour une école doctorale suisse de didactique qui devrait, notamment par l'encadrement des doctorants désireux d'écrire une thèse en didactique, permettre la formation de la relève, au niveau professoral, des Hautes Ecoles Pédagogiques et des Universités.

La rubrique discussion a ici pour visée de contextualiser les contributions de la partie thématique dans l'actualité scientifique et institutionnelle suisse et de montrer les principaux enjeux pour la recherche et la formation des enseignants que constituent la tertiairisation et l'universitarisation de cette formation.

Disons-le d'emblée, ce que nous avions envisagé n’a que partiellement réussi. Espérons que le présent numéro suscitera des contributions ultérieures, dans la partie Varia. Malgré notre volonté, dans le temps imparti par l'édition du présent numéro de la revue, nous n'avons pas obtenu une contribution du point de vue de la didactique générale. La restructuration de la recherche et de la formation en Suisse semble mobiliser les rares ressources existant dans le domaine didactique. 
Par ailleurs, si les deux contributions portant sur la didactique des langues premières, plus précisément sur la didactique de l'expression écrite (Bronckart, ici même) et la didactique de l'écriture (Sieber, ici même), représentent une mise en dialogue de l'évolution des recherches et des questions dans un champ proche, sur une période semblable, nos démarches du côté de la didactique des mathématiques en Suisse alémanique ou en Allemagne n'ont pas abouti. L'absence de répondant de la didactique des mathématiques s'explique de notre point de vue d'une part par la réorganisation de la formation des enseignants dans les structures des Hautes Études Pédagogiques et d'autre part certainement aussi par l'ancrage disciplinaire de la présente revue en sciences de l'éducation.

La mise en place des Hautes Études Pédagogiques suppose une redéfinition de la recherche entre institution de formation et Université. Eu égard au caractère récent de la restructuration, les chercheurs intéressés ont été objectivement limités dans leur possibilité de tracer une évolution de leur champ. Par ailleurs, les didacticiens se revendiquant d'un ancrage universitaire «mathématiques» disposent de leurs propres réseaux et ne voient pas un intérêt immédiat à publier une contribution dans une revue des sciences de l'éducation. Pour le développement du champ - pas seulement pour la didactique des mathématiques, la question de l'ancrage institutionnel des didactiques reste une question vive. Notonsle, les auteurs ayant contribué au présent numéro sont impliqués dans la formation des enseignants - ce qui n'est pas nouveau pour la didactique disciplinaire et générale -, en sciences humaines et sociales, à savoir dans leur champ disciplinaire et/ou en sciences de l'éducation.

Parmi les nombreux aspects susceptibles de montrer l'évolution du champ didactique, nous nous limitons à trois aspects. Un autre aspect doit alimenter les discussions à venir: le positionnement, institutionnel aussi, des didactiques disciplinaires en tension avec les sciences de l'éducation et sociales, les disciplines académiques et le champ scolaire (Fachkommission Fachdidaktik, 1998; Projekt Fachdidaktik, 2005). Nous avons retenu les trois aspects suivants:

1. Les standards de formation et les compétences attendues des élèves;

2. Le curriculum scolaire et la progression des contenus;

3. L'actualité de la prise en compte du triangle didactique: élève, contenu d'enseignement et enseignant.

1. Le débat sur l'impact de l'enseignement/apprentissage scolaire ou sur les capacités des élèves sortant du système de formation officiel est d'actualité; il interroge les didactiques dans leurs capacités d'intervention. Sont-elles à même de fournir des orientations et des contenus d'enseignement/apprentissage répondant aux aspirations et attentes politiques et sociales? Aujourd'hui, cette discussion s'organise à partir de l'élaboration de standards de formation permettant l'évaluation des élèves et la définition de niveaux de compétences susceptibles d'être testés. Est-ce que ces termes - standards et compétences - représentent des termes familiers aux didactiques? Comment les prennent-elles 
en charge? Orientées sur les capacités d'agir en situation et le résultat, sur quelles bases s'appuient l'enseignement/apprentissage de compétences? Comment s'agence la progression des contenus? Conformément au principe que ce qui s'évalue est enseigné, quel curriculum fonde le choix des tâches évaluées et des situations? Les deux dernières questions concernent le deuxième aspect retenu par nous.

2. Les aspirations et les attentes politico économico sociales à l'égard du système éducatif se manifestent non seulement du point de vue de l'impact et du résultat, mais aussi par une exigence de cohérence et de systématicité du point de vue des contenus d'enseignement transmis aux générations futures. Ce sont par l'agencement des contenus, leur organisation systématique et leur traduction en moyens d'enseignement dans un curriculum que s'opérationnalisent ces aspirations et attentes (Westbury, 1998). Destiné à orienter les pratiques des enseignants, le curriculum devrait fournir les conceptualisations susceptibles d'être traduites en plan d'action - en tâches, en situations, voire en séquences d'enseignement organisées dans une cohérence - pour doter les élèves des capacités attendues tout au long de la scolarité. Le curriculum devrait en conséquence répartir les contenus selon un agencement temporel, selon une progression, et anticiper les principaux apprentissages de façon à les échelonner de manière optimale. La recherche didactique ne peut se soustraire à cette réflexion d'ordre curriculaire. Programmatiquement, elle concerne tout le cursus scolaire. Est-ce que les différentes contributions entrent d'une manière ou d'une autre en matière sur cette réflexion?

3. Depuis leur émergence dans les années 1970-1980, les didactiques disciplinaires se sont revendiquées du triangle didactique, élève, contenu d'enseignement et enseignant. Privilégiant plutôt l'un ou l'autre des axes - l'axe contenu-élève, l'axe contenu-enseignant ou encore, toujours en tenant compte du contenu, l'axe enseignant-élève -, la prise en compte des trois termes suppose cependant de privilégier un point de vue. En regardant quels sont les axes prioritaires, le triangle didactique constitue une troisième manière d'observer l'évolution du champ didactique. Assiste-t-on à un déplacement des axes? Si oui, lequel ?

Lévolution que nous pouvons esquisser montre un déplacement de la réforme des programmes et d'une élaboration innovante des contenus vers des conceptualisations marquant une rupture avec la logique des réformes du système éducatif adoptant un point de vue d'innovation. Certaines didactiques vont jusqu'à s'intéresser à une analyse du fonctionnement ordinaire de l'enseignement/apprentissage d'un contenu donné et de ces contraintes générales et spécifiques (Margolinas; Schubauer-Leoni \& Leutenegger, ici même).

Au-delà de l'ancrage culturel des didactiques francophones ou germanophones, ces conceptualisations proprement didactiques consistent en emprunts effectués dans différentes disciplines académiques du point de vue des exigences 
et des contraintes de l'enseignement/apprentissage d'un contenu donné. Ce mouvement d'emprunts et de réélaboration, à caractère interdisciplinaire, finalisé par l'enseignement/apprentissage est présenté dans toutes les contributions de la partie thématique du présent numéro et se trouve pleinement théorisé comme phénomène didactique d'ensemble dans la contribution de SchubauerLeoni et Leutenegger "Une relecture des phénomènes transpositifs à la lumière de la didactique comparée». De ce point de vue, l'applicationnisme strict est pour le moins en voie de dépassement, puisque les emprunts se trouvent raisonnés et réélaborés dans une perspective didactique.

La référence aux compétences représente une illustration de cette élaboration propre aux didactiques. Terme revendiqué ou contesté dans les contributions de didactique des langues, le débat n'est abordé que par la bande. Emanant du monde du travail, visant l'adéquation à la situation et l'efficacité (Bronckart $\&$ Dolz, 2000; Bulea \& Bronckart, 2005), une compétence se définit de manière contradictoire, soit transversalement ou au contraire disciplinairement, soit en tant que ressources disponibles de l'agent ou en tant que processus dynamique au cours duquel les ressources sont mobilisées dans l'agir. Par exemple, la définition des compétences ${ }^{3}$ sur laquelle s'appuie le projet d' Harmo nisation de la s colarité obligatoire (HARMOS) (Conférence suisse des Directeurs cantonaux de l'Instruction publique, 2004), conformément à un principe d'éducabilité, désigne les ressources dont dispose l'individu ou qu'il peut acquérir. La compétence consiste en une description d'états distincts, selon des niveaux. HARMOS conçoit les compétences du point de vue des et en lien avec les fondements des disciplines scolaires. Pour être mesurables, les compétences sont traduites en tâches et en situations problèmes. Ce point de vue concerne indubitablement les didactiques dans la mesure où elles se proposent d'étudier les tâches, les situations, le milieu ou plus largement les séquences d'enseignement/apprentissage.

Une mise en veilleuse des interrogations curriculaires s'observe-t-elle? La contribution ici présente de Sieber pourrait le suggérer lorsqu'il évoque une période - dépassée - de recherche et de révision du curriculum. De l' input on serait passé à l' output ou mieux encore à l' outcome, c'est-à-dire non pas aux résultats, mais aux retombées de l'enseignement/apprentissage. De fait l'accent, relevé dans les contributions, mis sur les tâches et les situations, et l'analyse des conditions d'études des élèves peut aussi être vu comme un approfondissement faisant suite aux interrogations curriculaires interrogeant autrement la logique d'ensemble. La didactique pourrait-elle cependant se soustraire à la tâche de penser les progressions, échapper à l'étude de ce que produirait la juxtaposition des tâches et des situations d'écriture, mathématiques ou d'écoute musicale? Concevoir les contenus de manière cohérente pour l'ensemble de l'école obligatoire reste une perspective, d'ailleurs entamée, mais encore largement programmatique (voir Dolz \& Schneuwly, 1996; ou pour le cycle 4 à 8 ans, Saada-Robert, Claret Girard, Veuthey \& Rieben, 1997). Même si les tâches, les situations ou les séquences sont élaborées de manière à intégrer la complexité des processus en jeu 
dans les activités d'enseignement/apprentissage, l'agencement temporel des contenus opérationnalisés par le truchement de tâches, de situations ou de séquences constitue un défi pour les didactiques qui ne semble pas vraiment levé. La didactique des mathématiques appréhendent les phénomènes temporels au sein de la conduite d'une situation (Margolinas, ici même), mais comment conceptualise-t-elle les décisions relatives à l'ordre temporel concernant aussi bien la répartition des objectifs généraux entre les différents cycles de l'enseignement obligatoire (progression intercycle) que l'agencement temporel des objectifs et des contenus disciplinaires à l'intérieur de chaque cycle (progression intracycle)? La définition de compétences attendues visée par le projet HARMOS peut-elle se passer de telles élaborations? La question concerne toutes les didactiques disciplinaires.

Concernant le triangle didactique, les contributions le convoquent en privilégiant un des axes. L'entrée par les processus rédactionnels, la contribution de Sieber, porte sans conteste sur l'axe élève et l'activité d'écriture textuelle, dans ses composantes complexes. Il se trouve le seul parmi les contributeurs du numéro à explicitement adopter le point de vue de l'apprenant et du contenu en se revendiquant d'une compétence textuelle que l'école se doit d'enseigner. La contribution de Margolinas montre que cet axe a constitué l'axe privilégié dans les années 1980. Pour étudier les situations d'ingénierie didactique, le didacticien des mathématiques a assumé le rôle de l'enseignant pour se centrer sur l'axe contenuélève. Ce déplacement de responsabilité a engendré une rééquilibration vers l'axe contenu-enseignant.

Pour des raisons similaires, la didactique du français suit le même mouvement. Il s'agit d'explorer l'action de l'enseignant, c'est-à-dire d'observer quelles sont les conditions créées par l'enseignant, quelles sont les contraintes de l'enseignement et quels sont les contenus médiatisés au cours des séances en classe. Pour ce faire, la recherche didactique recourt à des méthodes d'observation empirique pour étudier les pratiques, soit, comme déjà précédemment, selon le point de vue de l'intervention didactique (Mili \& Rickenmann; Bronckart) soit en laissant l'enseignant élaborer lui-même le contenu, comme c'est le cas pour l'étude des pratiques enseignantes ordinaires (Margolinas; Schubauer-Leoni \& Leutenegger). Rompant avec la stricte logique d'intervention, un nouveau chantier, qui ne fait pas consensus, s'ouvre dès lors.

Les différentes contributions de la partie thématique et les trois aspects soulevés ici esquissent un vaste champ de réflexion. Le caractériser, identifier les convergences et les divergences en vue d'élaborer des perspectives pour le développement du champ didactique constitue la tâche de la rubrique discussion de B. Schneuwly. Plus généralement, espérons que la discussion aujourd'hui nécessaire s'entame et ne se verrouille pas. 


\section{Notes}

1 A noter que le terme de didactiques spécialisées est une appellation dans laquelle les didacticiens des disciplines francophones ne se reconnaissent pas et montre un découpage du champ en didactique générale et spécialisée.

2 Voir numéros d'Education et Recherche (3/1990 et 1/1991).

3 «Les standards de formation définissent en premier lieu les compétences que les établissements scolaires doivent développer chez leurs élèves afin que ceux-ci atteignent certains objectifs fondamentaux et précis de la formation, et ils déterminent en second lieu les compétences que les enfants et les jeunes doivent avoir acquis jusqu’à un degré scolaire précis. Troisièmement, ces compétences sont décrites suffisamment concrètement de sorte qu'elles puissent être traduites en tâches ou en situations-problèmes et puissent être saisies et vérifiées à l'aide de tests.» (CDIP, 2004, Finalités et conception du projet HARMOS, p. 7)

\section{Références bibliographiques}

Bronckart, J.-P. \& Dolz, J. (2000). La notion de compétence: quelle pertinence pour l'étude de l'apprentissage des actions langagières. In J. Dolz \& E. Ollagnier (Hrsg.), L'énigme de la compétence en éducation (Raisons Éducatives $\mathrm{n}^{\circ}$ 2, S. 27-44). Bruxelles: De Beock.

Bulea, E. \& Bronckart, J.-P. (2005). Coda: pour une approche dynamique des compétences (langagières). In J.-P. Bronckart, E. Bulea \& M. Pouliot (Hrsg.), Repenser l'enseignement des langues: comment identifier et exploiter les compétences (S. 193-227). Villeneuve d'Ascq: Presses universitaires du Septentrion.

Conférence suisse des Directeurs cantonaux de l'Instruction publique (CDIP) (2004). HARMOS. Finalités et conception du projet, juin 2004 . Berne: Secrétariat général.

Dolz, J. \& Schneuwly, B. (1996). Genres et progression en expression orale et écrite. Eléments de réflexions à propos d'une expérience romande. Enjeux, 37/38, 49-75.

Fachkommission Fachdidaktik (1998). Impulse für die Fachdidaktik in der Schweiz. Beiträge zur Lehrerbildung (BzL), 16/2, 222-230.

Projekt Fachdidaktik (2005). Fachdidaktik in der Schweiz-gezielter Kompetenzaufbau: Qualifizierung von Dozierenden und Etablierung der Forschung. Schlussbericht. September 2005. Bern: EDK/CRUS/SKPH.

Saada-Robert, M., Claret Girard, V., Veuthey, C. \& Rieben, L. (1997). Situations didactiques complexes et spécifiques pour l'entrée dans l'écrit en 1E-2E-1P-2P. Genève: FPSE et Maison des Petits.

Westbury, I. (1998). Didaktik and Curriculum Studies. In B. Gundem \& S. Hopmann, Didaktik and/or Currriculum: an International Dialogue (pp. 47-78). New York: Peter Lang. 
T h e m a 